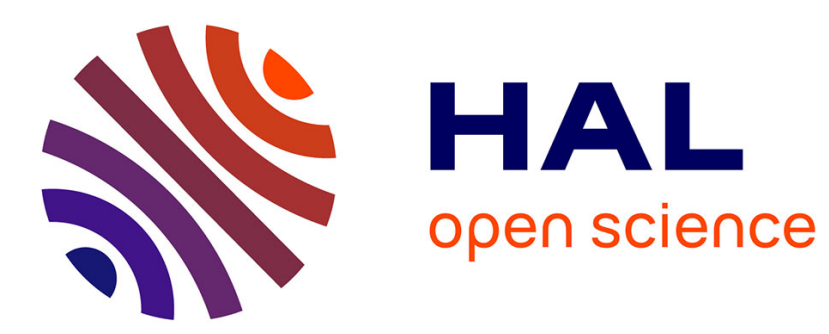

\title{
Finite-difference modeling of Biot's poroelastic equations across all frequencies
}

\author{
Yder J. Masson, Steven R Pride
}

\section{To cite this version:}

Yder J. Masson, Steven R Pride. Finite-difference modeling of Biot's poroelastic equations across all frequencies. Geophysics, 2010, 75 (2), pp.N33 - N41. 10.1190/1.3332589 . hal-01653918

\section{HAL Id: hal-01653918 \\ https://hal.science/hal-01653918}

Submitted on 5 Dec 2017

HAL is a multi-disciplinary open access archive for the deposit and dissemination of scientific research documents, whether they are published or not. The documents may come from teaching and research institutions in France or abroad, or from public or private research centers.
L'archive ouverte pluridisciplinaire HAL, est destinée au dépôt et à la diffusion de documents scientifiques de niveau recherche, publiés ou non, émanant des établissements d'enseignement et de recherche français ou étrangers, des laboratoires publics ou privés. 


\title{
Finite-difference modeling of Biot's poroelastic equations across all frequencies
}

\author{
Y. J. Masson ${ }^{1}$ and S. R. Pride ${ }^{2}$
}

\begin{abstract}
An explicit time-stepping finite-difference scheme is presented for solving Biot's equations of poroelasticity across the entire band of frequencies. In the general case for which viscous boundary layers in the pores must be accounted for, the time-domain version of Darcy's law contains a convolution integral. It is shown how to efficiently and directly perform the convolution so that the Darcy velocity can be properly updated at each time step. At frequencies that are low enough compared to the onset of viscous boundary layers, no memory terms are required. At higher frequencies, the number of memory terms required is the same as the number of time points it takes to sample accurately the wavelet being used. In practice, we never use more than 20 memory terms and often considerably fewer. Allowing for the convolution makes the scheme even more stable (even larger time steps might be used) than it is when the convolution is entirely neglected. The accuracy of the scheme is confirmed by comparing numerical examples to exact analytic results.
\end{abstract}

\section{INTRODUCTION}

In a recent work, Masson et al. (2006) present a simple time-stepping staggered-grid finite-difference scheme for solving Biot's (1956) equations of wave propagation in porous materials. They restrict their analysis to seismic frequencies that are low enough so that the generation of viscous boundary layers in the pores of the rocks can be neglected. For consolidated earth materials such as sandstones, the transition frequency at which viscous boundary layers first develop is typically greater than $100 \mathrm{kHz}$, so schemes that neglect this physics are valid for most seismic applications. Accordingly, most studies that present finite-difference approaches for solving Biot's equations (e.g., Zhu and McMechan, 1991; Carcione and
Quiroga-Goode, 1995; Özdenvar and McMechan, 1997; Carcione and Helle, 1999; and Zhang, 1999) focus on the low-frequency form of the equations.

However, in unconsolidated sediments, the transition frequency at which viscous boundary layers must be accounted for can be as small as $1 \mathrm{kHz}$ (or even less). Therefore, for many seismological applications to unconsolidated (or high-permeability) sediments, it is useful to have a finite-difference scheme for solving Biot's equations across the entire band of frequencies. Furthermore, many laboratory experiments on porous materials are conducted at ultrasonic frequencies, in which case it is always necessary to account for the development of viscous boundary layers. In poroelastic theory, such pore-scale dynamics is allowed for in the time domain by using a "dynamic-permeability" convolution operator in a generalized Darcy's law. In the frequency domain, the dynamic permeability corresponds to a complex frequency-dependent permeability coefficient.

Carcione (1996) presents a finite-differencing approach that allows for the dynamic permeability by approximating it as a sum of Zener relaxation functions. It is of interest to more directly treat the explicit time-domain form of the dynamic-permeability convolution operator using finite differences. To this end, Hanyga and Lu (2005) first convert the convolution integral to an integral over an infinite time domain and then implement a somewhat complicated application of the Gauss-Jacobi and Laguerre quadrature formulas. The present study presents a more direct evaluation of the dynamic-permeability convolution and requires no more memory terms to perform the convolution than it takes to cover the waveform with discretization points in time. Its advantage thus is being both efficient and relatively simple to implement.

Using the classic work of Levander (1988) on the elastodynamic equations as our guide, we present our poroelastic finite-differencing scheme in two dimensions instead of three for reasons of compactness and clarity. Taking the algorithm to three dimensions involves adding another spatial loop within the time loop and writing down the update equations for all of the tensorial components.

Manuscript received by the Editor 17 April 2009; revised manuscript received 15 August 2009; published online 12 March 2010.

${ }^{1}$ University of California at Berkeley, Department of Earth and Planetary Sciences, Berkeley, California, U.S.A. E-mail: yder_masson@ berkeley.edu.

${ }^{2}$ Lawrence Berkeley National Laboratory, Earth Sciences Division, Berkeley, California, U.S.A.E-mail: srpride@ @ lbl.gov.

(C) 2010 Society of Exploration Geophysicists. All rights reserved. 


\section{POROELASTIC RESPONSE}

Poroelasticity not only accounts for the displacements and stresses acting on each voxel of a porous body, but allows for the fluidpressure changes and fluid flow as well. Implicit in the theory is that the wavelength of a mechanical disturbance moving through a porous material is far greater than the size of the grains making up the material so that a porous-continuum description is justified.

The fluid flow is well modeled using a generalized Darcy's law that allows for flow due to induced pressure gradients and for flow created by the acceleration of the framework of grains, which is the frame of reference for the relative fluid motion. Assuming an $e^{-i \omega t}$ time dependence, the generalized Darcy's law is written in the frequency domain as

$$
\mathbf{q}=\frac{k(\omega)}{\eta}\left[-\nabla p+i \omega \rho_{f} \mathbf{v}\right] .
$$

Here, $p$ is the fluid pressure, $\mathbf{q}$ the Darcy filtration velocity, $\mathbf{v}$ the velocity of the solid framework of grains, $\eta$ the fluid viscosity, $\rho_{f}$ the fluid density, and $k(\omega)$ the complex (or "dynamic") permeability.

The frequency dependence in $k(\omega)$ results from the appearance of viscous boundary layers in the pores at sufficiently high frequencies. At low frequencies, the flow in each pore is controlled by viscous shearing and is entirely laminar. At high frequencies, inertial effects begin to dominate the shear forces, resulting in an ideal "plug flow" in each pore except near the fluid/solid interface where shear forces again must dominate because the relative motion is zero on the grain surfaces. Thus, there are created viscous boundary layers near the grain surfaces whose thickness decrease with increasing frequency as $1 / \sqrt{\omega}$.

Johnson et al. (1987) derive a complex permeability function that connects these two frequency limits while obeying causality constraints. Their model for the frequency dependence of $k(\omega)$ is

$$
\frac{k(\omega)}{k_{0}}=\left[\sqrt{1-i \frac{\omega}{\Omega}}-i \frac{\omega}{\omega_{J}}\right]^{-1},
$$

where the two relaxation frequencies $\omega_{J}$ and $\Omega$ are defined as

$$
\begin{gathered}
\omega_{J}=\frac{\eta}{\rho_{f} F k_{0}} \\
\Omega=\frac{n_{J} \omega_{J}}{4},
\end{gathered}
$$

with $n_{J}$ a dimensionless parameter given by

$$
n_{J}=\frac{\Lambda^{2}}{F k_{0}} .
$$

Here, $k_{0}$ is the steady-flow (zero frequency) limit of the permeability, $F$ is the electrical formation factor, and $\Lambda$ is a weighted pore-volume to grain-surface ratio with the weight emphasizing constricted portions of the pore space (see Johnson et al., 1987, for the precise mathematical definition of $\Lambda$ ) that is also an important length parameter in modeling the surface electrical conductivity in rocks (Pride, 1994). For clean sands, $n_{J}=8$ is consistent with both numerical and laboratory experiments. For shaly sands, one can have $n_{J} \ll 8$. Physically, $\Omega$ is the circular frequency at which viscous boundary layers first develop.
We perform the finite-difference modeling in the time domain. Plyushchenkov and Turchaninov (2000) analytically obtain the inverse Fourier transfom of the $k(\omega)$ given by equation 2 . Using this result, the time-domain version of the generalized Darcy's law in equation 1 is exactly equivalent to

$$
\begin{aligned}
& -\nabla p-\rho_{f} \frac{\partial \mathbf{v}}{\partial t}=\rho_{f} F \frac{\partial \mathbf{q}}{\partial t}+\frac{\eta}{k_{0}} \int_{0}^{t} \frac{e^{-\Omega(t-s)}}{\sqrt{\pi \Omega(t-s)}} \\
& \times\left[\frac{\partial \mathbf{q}(s)}{\partial s}+\Omega \mathbf{q}(s)\right] d s,
\end{aligned}
$$

where $s$ is the past time variable. This result is easily confirmed by taking the Fourier transform of equation 6 and using the convolution theorem to obtain equations 1 and 2 (going the other direction is more involved). Upon taking the leading order in $-i \omega$ low-frequency limit in equation 2 and then returning to the time domain, one obtains the low-frequency variant of equation 6:

$$
-\nabla p-\rho_{f} \frac{\partial \mathbf{v}}{\partial t}=\left(1+\frac{2}{n_{J}}\right) \rho_{f} F \frac{\partial \mathbf{q}}{\partial t}+\frac{\eta}{k_{0}} \mathbf{q} .
$$

Our earlier work on how to perform finite-difference modeling of the Biot equations (Masson et al., 2006) was based on the low-frequency law of equation 7 . The present work deals with the entire frequency range and specifically addresses how to introduce the convolution of equation 6 into the scheme.

The remaining equations of Biot's theory include the total conservation of linear momentum

$$
\rho \frac{\partial \mathbf{v}}{\partial t}=\nabla \cdot \boldsymbol{\tau}-\rho_{f} \frac{\partial \mathbf{q}}{\partial t},
$$

where $\rho$ is the bulk density of the porous material and $\tau=\tau_{i j} \hat{\mathbf{i}} \hat{\mathbf{j}}$ is the total stress tensor, as well as the stress-strain constitutive laws for an isotropic porous material

$$
\begin{gathered}
\frac{\partial \boldsymbol{\tau}}{\partial t}=\left(\lambda_{u} \nabla \cdot \mathbf{v}+\alpha M \nabla \cdot \mathbf{q}\right) \mathbf{I}+\mu\left[\nabla \mathbf{v}+(\nabla \mathbf{v})^{\mathbf{T}}\right] \\
-\frac{\partial p}{\partial t}=M(\alpha \nabla \cdot \mathbf{v}+\nabla \cdot \mathbf{q})
\end{gathered}
$$

with $\mathbf{I}=\delta_{i j} \hat{\mathbf{i}} \hat{\mathbf{j}}$ the identity tensor. The poroelastic constants used here are the undrained Lamé modulus $\lambda_{u}$, the shear modulus $\mu$ (the same for drained and undrained conditions), the so-called Biot-Willis (1957) constant $\alpha$, and the fluid-storage coefficient $M$. Modeling suggestions and further discussion of these poroelastic constants are given in many places, including Pride (2005) and Masson et al. (2006).

\section{FINITE-DIFFERENCING SCHEME}

The time-stepping finite-difference scheme of Masson et al. (2006) for solving the low-frequency Biot (1956) equations is quite similar to Levander's (1988) 2D fourth-order velocity-stress staggered-grid method for the elastodynamic equations. Levander's (1988) scheme built on the velocity-stress staggered-grid modeling of Madariaga (1976) and Virieux (1986). 
The present work adopts the 2D velocity-stress staggered grid defined in Figure 1. The stress components $\tau_{x x}, \tau_{z z}$, and $p$ are assigned to the grid points $x=m \Delta_{x}, z=n \Delta_{z}$, where $m$ and $n$ are integers; the horizontal velocities $v_{x}$ and $q_{x}$ to the points $x=(m+1 / 2) \Delta_{x}, z$ $=n \Delta_{z}$; the vertical velocities $v_{z}$ and $q_{z}$ to the points $x=m \Delta_{x}, z=(n$ $+1 / 2) \Delta_{z}$; and the shear stress $\tau_{x z}$ to the points $x=(m+1 / 2) \Delta_{x}, z$ $=(n+1 / 2) \Delta_{z}$. Further, all the velocities are temporally discretized at the time points $t=l \Delta_{t}$, whereas all the stresses are discretized at the time points $t=(l+1 / 2) \Delta_{t}$

Any order of differencing approximation could be used for the first-space derivative operators $D_{x}$ and $D_{z}$ and the first-time derivative $D_{t}$ in what follows. However, the stability analysis and numerical implementation of the present study use the second-order time derivative and fourth-order space operator given by

$$
\begin{aligned}
\left.D_{x} v_{x}\right|_{m, n}= & \frac{1}{\Delta_{x}}\left\{c_{1}\left[v_{x}(m+1 / 2, n)-v_{x}(m-1 / 2, n)\right]\right. \\
& \left.-c_{2}\left[v_{x}(m+3 / 2, n)-v_{x}(m-3 / 2, n)\right]\right\},
\end{aligned}
$$

with $c_{1}=9 / 8$ and $c_{2}=1 / 24$ the fourth-order differencing weights.

\section{Update equations for stresses and pressure}

Knowing $q_{i}, v_{i}$ at time $t=l \Delta_{t}$ and $\tau_{i j}, p$ at time $t=(l-1 / 2) \Delta_{t}$, the discrete form of the constitutive laws 9 and 10 are used to update $\tau_{i j}$ and $p$ at time $t=(l+1 / 2) \Delta_{t}:$

$D_{t} \tau_{x x}=\left(\lambda_{u}+2 \mu\right) D_{x} v_{x}+\lambda_{u} D_{z} v_{z}+\left.\alpha M\left(D_{x} q_{x}+D_{z} q_{z}\right)\right|_{m, n, l}$

$D_{t} \tau_{z z}=\lambda_{u} D_{x} v_{x}+\left(\lambda_{u}+2 \mu\right) D_{z} v_{z}+\left.\alpha M\left(D_{x} q_{x}+D_{z} q_{z}\right)\right|_{m, n, l}$

$$
D_{t} \tau_{x z}=\left.\mu\left(D_{x} v_{z}+D_{z} v_{x}\right)\right|_{m+\frac{1}{2}, n+\frac{1}{2}, l}
$$

$$
D_{t} p=-\alpha M\left(D_{x} v_{x}+D_{z} v_{z}\right)+\left.M\left(D_{x} q_{x}+D_{z} q_{z}\right)\right|_{m, n, l}
$$

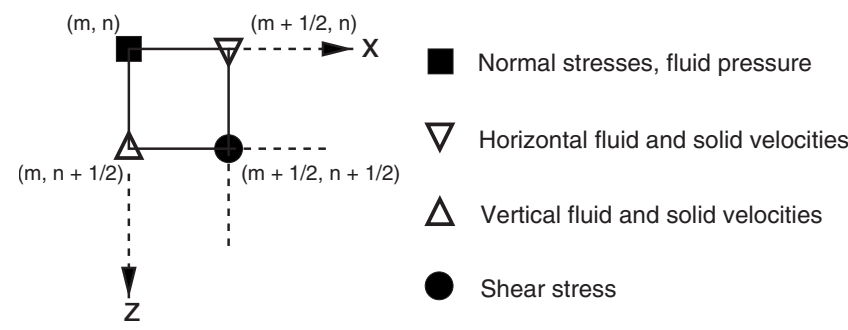

Figure 1. Convention used for the spatial position of the stresses, pressure, and fluid and solid velocities on the staggered grid. where $D_{t}, D_{x}$, and $D_{z}$ denote finite-difference derivatives and where the vertical line at the right of each equation denotes the space and time position at which all of the terms in the equations are centered.

\section{Update equations for the Darcy velocity}

To treat the convolution in equation 6 , the integration domain is broken into a finite number $N+1$ of past time intervals. The number $N$ can be chosen so that $e^{-\Omega N \Delta_{t}}<\epsilon$ or

$$
N=-\frac{\ln \epsilon}{\Omega \Delta_{t}},
$$

where $\epsilon$ is a small number like $10^{-3}$ that determines the accuracy.

To perform the integrations, we assume that within each of the past finite-difference time intervals $\Delta_{t}$, the Darcy velocity is continuously varying as a linear function. As Figure 2 indicates, the first time interval is half $\Delta_{t}$, whereas each of the remaining $j=1, N$ intervals are of duration $\Delta_{t}$. We have

$$
I(t)=\int_{0}^{t} d s \frac{e^{-\Omega(t-s)}}{\sqrt{\pi \Omega(t-s)}}\left[\frac{\partial q(s)}{\partial s}+\Omega q(s)\right]
$$

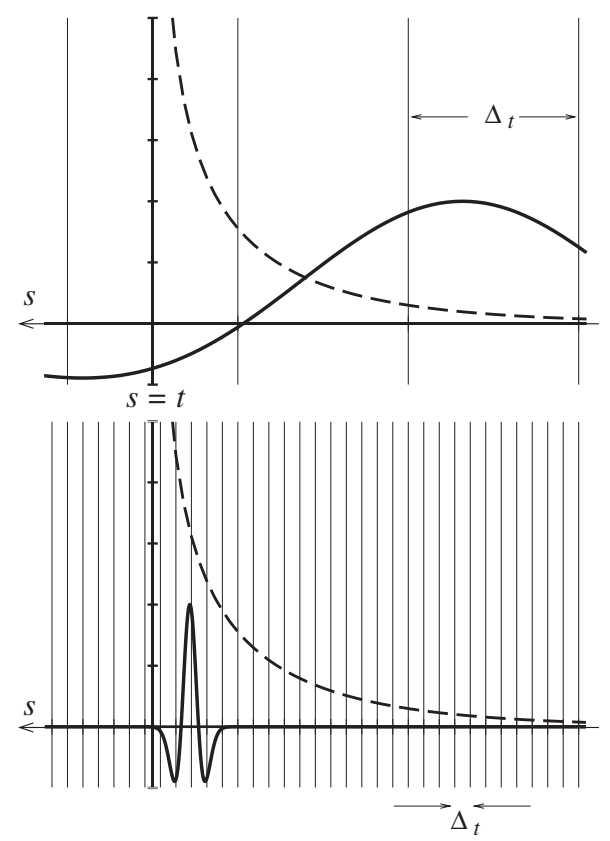

Figure 2. Schematic of the kernel of the convolution integral (dashed-line curves, normalized to be unitless) and Darcy flow (solid curves, normalized to be unitless) as a function of the convolution time variable $s$. The upper part corresponds to seismic frequencies that are smaller than the viscous-boundary-layer transition $\left(\Omega \Delta_{t}\right.$ $>1)$. The lower part corresponds to seismic frequencies that are larger than the viscous-boundary-layer transition $\left(\Omega \Delta_{t}<1\right)$. Present time corresponds to $s=t$ where the kernel has an integrable singularity. 


$$
\begin{aligned}
& \approx \int_{t-\Delta_{t} / 2}^{t} d s \frac{e^{-\Omega(t-s)}}{\sqrt{\pi \Omega(t-s)}}\left\{\frac{\partial q}{\partial t}+\Omega\left[q(t)+(s-t) \frac{\partial q}{\partial t}\right]\right\} \\
& \quad+\sum_{j=1}^{N} \int_{t-j \Delta_{t}-\Delta_{t} / 2}^{t-j \Delta_{t}+\Delta_{t} / 2} d s \frac{e^{-\Omega(t-s)}}{\sqrt{\pi \Omega(t-s)}}\left\{\left.\frac{\partial q}{\partial s}\right|_{t-j \Delta_{t}}\right. \\
& \left.+\Omega\left[\left.q\right|_{t-j \Delta_{t}}+\left.\left(s-t+j \Delta_{t}\right) \frac{\partial q}{\partial s}\right|_{t-j \Delta_{t}}\right]\right\} .
\end{aligned}
$$

In the final line of equation 18 , both $q(s)$ and its time derivative $\partial q(s) / \partial s$ are being evaluated at the time $s=t-j \Delta_{t}$ in the center of each interval $j$ and are therefore constants in each interval that can be taken outside the integrals. For $N$ sufficiently large, the only approximation in passing from equation 17 to 18 is taking the Darcy velocity as linearly varying in each time interval $\Delta_{t}$.

If the current finite-difference time index for the Darcy velocity is $l$, the current time in the convolution integral of equation 18 is $t=(l$ $+1 / 2) \Delta_{t}$. Upon making the substitution of variables $u=\Omega(t-s)$ in the above integrals, the discrete form of equation $18 \mathrm{can}$ be written as

$$
\begin{aligned}
I\left(l+\frac{1}{2}\right)= & \frac{\left(G_{o}-H_{o}\right)}{\Omega} D_{t} q+G_{o}\langle q\rangle \\
& +\sum_{j=1}^{N}\left\{\frac{\left[\left(1+j \Omega \Delta_{t}\right) G_{j}-H_{j}\right]}{\Omega} D_{t} q\right. \\
& \left.+G_{j}\langle q\rangle\right\}\left.\right|_{l+\frac{1}{2}-j}
\end{aligned}
$$

where the coefficients $G_{o}, H_{o}, G_{j}$, and $H_{j}$ are defined as

$$
\begin{gathered}
G_{o}=\int_{0}^{\Omega \Delta_{t} / 2} \frac{e^{-u}}{\sqrt{\pi u}} d u \\
G_{j}=\int_{(j-1 / 2) \Omega \Delta_{t}} \frac{e^{-u}}{\sqrt{\pi u}} d u \\
H_{o}=\int_{0}^{(j+1 / 2) \Omega \Delta_{t} / 2} e^{-u} \sqrt{\frac{u}{\pi}} d u \\
H_{j}=\int_{(j+1 / 2) \Omega \Delta_{t}} e^{-u} \sqrt{\frac{u}{\pi}} d u,
\end{gathered}
$$

and where the second-order discrete time derivative $D_{t} q$ and the average $\langle q\rangle$ when centered at $l+1 / 2$ are defined as

$$
D_{t} q=\frac{q(l+1)-q(l)}{\Delta_{t}}
$$

$$
\langle q\rangle=\frac{q(l+1)+q(l)}{2} .
$$

The coefficients $G_{o}, H_{o}, G_{j}$, and $H_{j}$ in equations 20-23 depend on only the material property $\Omega$ and the time interval $\Delta_{t}$. They can be computed ahead of time using any favorite integral solver. For finite limits on the integrals, they cannot be computed analytically. However, in the limit that $\Omega \Delta_{t} \gg 1$, which would correspond to the lowfrequency seismic limit for wave propagation applications, we have the analytic results that $G_{o}=1, H_{o}=1 / 2$, and $G_{j}=H_{j}=0$.

Using these results for the convolution, and inserting the discrete form of equation 8 into the discrete form of equation 6 , gives the update equations for determining $q_{i}(l+1)$ :

$$
\begin{gathered}
{\left[\psi+\frac{\eta}{k_{0}} \frac{\left(G_{o}-H_{o}\right)}{\Omega}\right] D_{t} q_{x}+\frac{\eta}{k_{0}} G_{o}\left\langle q_{x}\right\rangle=-\frac{\eta}{k_{0}} S_{x}-D_{x} p} \\
\quad-\left.\frac{\rho_{f}}{\rho}\left(D_{x} \tau_{x x}+D_{z} \tau_{x z}\right)\right|_{m+\frac{1}{2}, n, l+\frac{1}{2}} \\
{\left[\psi+\frac{\eta}{k_{0}} \frac{\left(G_{o}-H_{o}\right)}{\Omega}\right] D_{t} q_{z}+\frac{\eta}{k_{0}} G_{o}\left\langle q_{z}\right\rangle=-\frac{\eta}{k_{0}} S_{z}-D_{z} p} \\
-\left.\frac{\rho_{f}}{\rho}\left(D_{z} \tau_{z z}+D_{x} \tau_{x z}\right)\right|_{m, n+\frac{1}{2}, l+\frac{1}{2}}
\end{gathered}
$$

Here, the parameter $\psi$ is defined as

$$
\psi=\rho_{f} F-\frac{\rho_{f}^{2}}{\rho},
$$

and $S_{x}$ and $S_{z}$ are defined as

$$
\begin{aligned}
S_{x}= & \sum_{j=1}^{N}\left\{\frac{\left[\left(1+j \Delta_{t} \Omega\right) G_{j}-H_{j}\right]}{\Omega} D_{t} q_{x}\right. \\
& \left.+G_{j}\left\langle q_{x}\right\rangle\right\}\left.\right|_{m+\frac{1}{2}, n, l+\frac{1}{2}-j} \\
S_{z}= & \sum_{j=1}^{N}\left\{\frac{\left[\left(1+j \Delta_{t} \Omega\right) G_{j}-H_{j}\right]}{\Omega} D_{t} q_{z}\right. \\
& \left.+G_{j}\left\langle q_{z}\right\rangle\right\}\left.\right|_{m+\frac{1}{2}, n, l+\frac{1}{2}-j}
\end{aligned}
$$

and are the contributions to the convolution that come from the $N$ time steps that precede the first half time step. The dominant contribution to the convolution comes from the first half time step and is allowed for on the left-hand side of equations 26 and 27 by the terms involving $G_{o}$ and $H_{o}$.

In the limit of low seismic frequencies, or more specifically when $\Omega \Delta_{t} / 2 \gg 1$, we have $S_{x}=S_{z}=0, G_{o}=1$, and $H_{o}=1 / 2$; and it is easily verified that equations 26 and 27 exactly reduce to the low-frequency form of equation 7. In this limit, the kernel of the convolution is concentrated in the first half time interval of past time and effectively acts as a Dirac delta function so that no memory terms need to be kept (i.e., $N=0$ ). 
As seismic frequencies increase, $\Omega \Delta_{t}$ decreases and more memory terms must be stored to compute the convolution accurately as the simple rule $N=-\ln \epsilon /\left(\Omega \Delta_{t}\right)$ suggests. However, once $\Omega \Delta_{t}<1$, it is not necessary to keep increasing the number of memory terms indefinitely. In such a high-frequency limit, the lower part of Figure 2 illustrates how the seismic wavelet becomes more concentrated in time relative to the extent of the kernel. The convolution is important in this limit only when the wavelet is close to the present time, so one needs to keep only enough memory terms to cover the temporal extent of the wavelet. Accordingly, for central wave frequencies $\omega$ that satisfy $\omega>\Omega$ (the "high-frequency" domain), one needs to keep only $N=-\ln \epsilon /\left(\omega \Delta_{t}\right)$ past time points to obtain accurate results for the convolution. In practice, we never need to keep more than roughly 20 terms in memory.

\section{Update equations for the particle velocity}

Finally, knowing $\tau_{i j}, p$, and $D_{t} q_{i}$ at time $t=(l+1 / 2) \Delta_{t}$ and $v_{i}$ at time $t=l \Delta_{t}, v_{i}$ is updated at time $t=(l+1) \Delta_{t}$ by inserting equation 6 into equation 8 to obtain

$$
\begin{aligned}
& \rho D_{t} v_{x}=D_{x} \tau_{x x}+D_{z} \tau_{x z}-\left.\rho_{f} D_{t} q_{x}\right|_{m+\frac{1}{2}, n, l+\frac{1}{2}} \\
& \rho D_{t} v_{z}=D_{x} \tau_{x z}+D_{z} \tau_{z z}-\left.\rho_{f} D_{t} q_{z}\right|_{m, n+\frac{1}{2}, l+\frac{1}{2}} .
\end{aligned}
$$

Equations 12-32 provide our finite-difference modeling algorithm.

\section{STABILITY}

To investigate the stability of the numerical scheme, we first perform a von Neumann stability analysis in the case for which the convolution product in equations 26 and 27 is entirely neglected, i.e., an analysis assuming $\eta / k_{0}=0$. Then the effects of the remaining parameters on the stability $\left(\eta / k_{0} \neq 0\right.$ and $\left.N \neq 0\right)$ are tested numerically. It is demonstrated that stability always is achieved using the criterion in which $\eta / k_{0}$ is neglected. In passing, we note that in our earlier study (Masson et al., 2006), we performed a more complicated version of the analysis assuming that $\eta / k_{0} \neq 0$.

To keep the analytic treatment tractable, we consider a plane longitudinal disturbance advancing in the $x$-direction through a homogeneous material (i.e., $q_{z}, v_{z}, \tau_{z z}$, and $\tau_{x z}$ are set to zero along with all spatial derivatives with respect to $z$ ). To investigate stability in higher dimensions, we perform purely numerical tests to establish a criterion (see the discussion at the end of this section). Displacements $u_{x}$ and $w_{x}$ are introduced through the defining relations

$$
\begin{aligned}
& v_{x}=D_{t} u_{x} \\
& q_{x}=D_{t} w_{x} .
\end{aligned}
$$

In this case, the set of difference equations 12-32 can be combined into the matrix system

$$
Q u=0,
$$

where the $2 \times 2$ matrix operator $Q$ is given by

$$
Q=\left[\begin{array}{l|l}
\left(\lambda_{u}+2 \mu\right) D_{x x}-\rho D_{t t} & \alpha M D_{x x}-\rho_{f} D_{t t} \\
\hline \alpha M D_{x x}-\rho_{f} D_{t t} & M D_{x x}-\rho_{f} F D_{t t}
\end{array}\right]
$$

and

$$
u=\left[u_{x}, w_{x}\right]^{T} .
$$

Here, $D_{x x}$ and $D_{t t}$ are the finite second-derivative operators in space (fourth order) and time (second order), respectively.

The von Neumann stability analysis assumes that the independent solutions of equation 35 are of the form

$$
\left[\begin{array}{c}
u_{x}(m, l) \\
w_{x}(m, l)
\end{array}\right]=e^{i k m \Delta_{x}-i \omega \Delta_{t}}\left[\begin{array}{c}
u_{o} \\
w_{o}
\end{array}\right],
$$

where $k$ is a real spatial wavenumber. In this context, testing the stability of the numerical scheme is equivalent to testing the hypothesis

$$
\operatorname{Im}\{\omega\} \leq 0 \forall k .
$$

If equation 39 is true, then the scheme is stable.

An expression for the stability criterion is obtained by requiring the determinant of the linear system to vanish; i.e.,

$$
\operatorname{det}\left|Q e^{i k m \Delta_{x}-i \omega \Delta_{t}}\right|=0 .
$$

The two roots of equation 40 are

$$
D_{x x} e^{i k m \Delta_{x}-i \omega \Delta_{t}}=\frac{\pi_{2} \pm \sqrt{\pi_{2}^{2}-4 \pi_{3} \pi_{1}}}{2 \pi_{3}} D_{t t} e^{i k m \Delta_{x}-i \omega \Delta_{t}},
$$

where the $\pi_{i}$ coefficients are defined as

$$
\begin{gathered}
\pi_{1}=\rho_{f} \rho\left(F-\rho_{f} / \rho\right) \\
\pi_{2}=\rho_{f} F\left(\lambda_{u}+2 \mu\right)+\rho M-2 \alpha M \rho_{f} \\
\pi_{3}=M\left(\lambda_{u}+2 \mu-\alpha^{2} M\right) .
\end{gathered}
$$

It is easily established that the second-order finite-difference time derivatives yield

$$
D_{t t} e^{i k m \Delta_{x}-i \omega \Delta_{t}}=-\frac{4}{\Delta_{t}^{2}} \sin ^{2}\left(\frac{\omega \Delta t}{2}\right) e^{i k m \Delta_{x}-i \omega \Delta_{t}},
$$

whereas the fourth-order finite-difference space derivatives give

$$
D_{x x} e^{i k m \Delta_{x}-i \omega \Delta_{t}}=-\frac{4 \phi_{k}}{\Delta_{x}^{2}} e^{i k m \Delta_{x}-i \omega \Delta_{t}},
$$

with the periodic function $\phi_{k}$ given by

$$
\begin{aligned}
\phi_{k}= & \left\{c_{1}^{2}+2 c_{1} c_{2}\left[1-4 \cos ^{2}\left(\frac{k \Delta_{x}}{2}\right)\right]\right\} \sin ^{2}\left(\frac{k \Delta_{x}}{2}\right) \\
& +c_{2}^{2} \sin ^{2}\left(\frac{3 k \Delta_{x}}{2}\right) .
\end{aligned}
$$

Again, $c_{1}=9 / 8$ and $c_{2}=1 / 24$ are the fourth-order differencing weights.

The stability criterion is established by inserting equations 45 and 46 into equation 41 , and requiring that $\omega$ be real (i.e., $\operatorname{Im}\{\omega\}=0$ ). Taking the more restrictive solution that is associated with the minus sign in equation 41 , and using the maximum of the function $\phi_{k}$ with respect to $k$ [i.e., $\max \left\{\phi_{k}\right\}=\left(c_{1}+c_{2}\right)^{2}$ ] so that the restriction on $\Delta_{t}$ given $\Delta_{x}$ is as strong as possible, defines the domain in which the numerical scheme is stable when $\eta / k_{0}=0$ : 


$$
\Delta_{t} \leq \Delta_{x} \sqrt{\frac{\pi_{2}-\sqrt{\pi_{2}^{2}-4 \pi_{3} \pi_{1}}}{2\left(c_{1}+c_{2}\right)^{2} \pi_{3}}} .
$$

For all values of $F \leq \rho_{f} / \rho$, the algorithm is unconditionally unstable. Another way to see (and say) the same thing is to note that the coefficient $\psi=F-\rho_{f} / \rho$ present in equations 26 and 27 must be positive for the response to be stable.

Finally, an asymptotic analysis of the right-hand side of equation 48 as the parameter $F$ becomes large results in the linear relation between $\Delta_{x}$ and $\Delta_{t}$ asymptoting to the classic Courant condition that, in one dimension, is given by

$$
\Delta_{t} \leq \frac{\Delta_{x}}{\left(c_{1}+c_{2}\right) V_{\mathrm{P}}} .
$$

Here, $V_{\mathrm{P}}$ is the velocity associated with the undrained fast $\mathrm{P}$-wave

$$
V_{\mathrm{P}}=\sqrt{\frac{\lambda_{u}+2 \mu}{\rho}} .
$$

For typical values of $F$ in rocks, the stability requirement of equation 49 always applies.

We now test the effect of having a nonzero value for $\eta / k_{0}$ and account for any number $N$ of memory terms in equations 26 and 27. This true stability criterion is obtained by numerically implementing the full scheme for different values of $\Delta t$ and verifying stability for each $\Delta_{t}$. In Figure 3, we show the effect of adding more memory terms to equations 26 and 27 for a given value of $\eta / k_{0}$. When no memory terms are used, the stability citerion is the one in equation 48. Adding more memory terms tends to improve the stability until it reaches a plateau value somewhere between the analytic criterion in equation 48 and the classic Courant condition in equation 49. Note that the plateau is reached when $N$ is given by equation 16 and the convolution is being computed properly.

To see why allowing for the convolution makes the scheme even more stable (i.e., allows a larger time step to be used) compared to

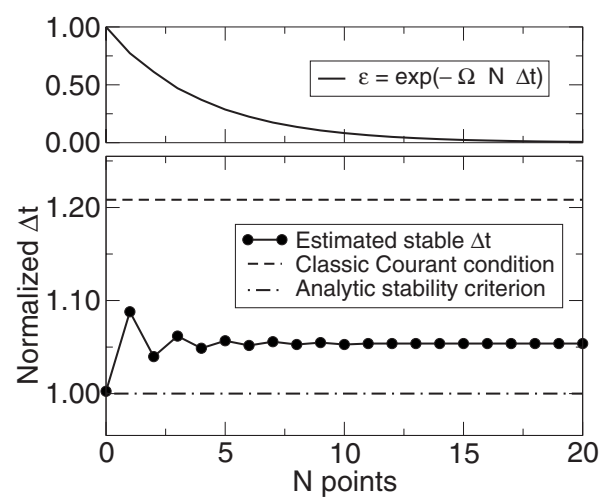

Figure 3. Evolution of the numerical stability of the scheme as a function of the number $N$ of memory variables used in equations 26 and 27. Top part: Percentage of the integral in equation 19 contained in the first $N$ terms of the sum. Bottom part: Stability criterion plotted as a function of the number of memory variables $N$ with $\eta / k_{0}$ $=$ const. When $N$ equals zero, the stability criterion is equal to the analytic criterion in equation 48 . When adding more memory variables, the stability criterion converges toward a plateau somewhere between the analytic criterion and the classic Courant value. Note that using an odd number of memory variables tends to stabilize the numerical scheme. the most restrictive condition of equation 48 , one needs to consider only the coefficient multiplying the time derivative of the Darcy velocity in equations 26 and 27 that we might call the "effective fluid inertia" and is given by $\psi+\left(G_{o}-H_{o}\right) \eta / k_{0}$. The convolution coefficients $G_{o}$ and $H_{o}$ (note that $G_{o}>H_{o}$ ) are adding to $\psi$ to make the effective inertia even larger than it was when the convolution and $\eta / k_{0}$ were neglected. Indeed, the more general condition for the scheme to be stable is that

$$
\psi>-\frac{\eta}{k_{0}} \frac{\left(G_{o}-H_{o}\right)}{\Omega},
$$

which is more strongly satisfied than the condition $\psi>0$ associated with equation 48 . Earth materials always have $\psi>0$, so this condition is always met.

In Figure 4, we present the behavior of the stability criterion as a function of $\eta / k_{0}$. The most important result is that the stability criterion is strictly increasing with $\eta / k_{0}$ (for the same reason that the effective fluid inertia is increasing with increasing $\eta / k_{0}$ ), which means that the criterion of equation 48 holds true for any value of $\eta / k_{0}$. The true stability limit for $\Delta_{t}$ is seen to lie somewhere between the criteria given by equations 48 and 49 .

Last, we have performed numerical tests of stability in 2D and 3D versions of the scheme and have determined empirically that dividing the right-hand side of equation 48 by $\sqrt{d}$, where $d$ is the Euclidian dimension of the modeling domain, gives an appropriate criterion. This is also the result for staggered-grid implementation of the elastodynamic equations (Virieux and Madariaga, 1982).

\section{NUMERICAL EXAMPLES}

In this section, we first present some numerical snapshots of the fields to demonstrate that the scheme produces qualitatively reasonable (expected) results. We then go on to demonstrate the accuracy

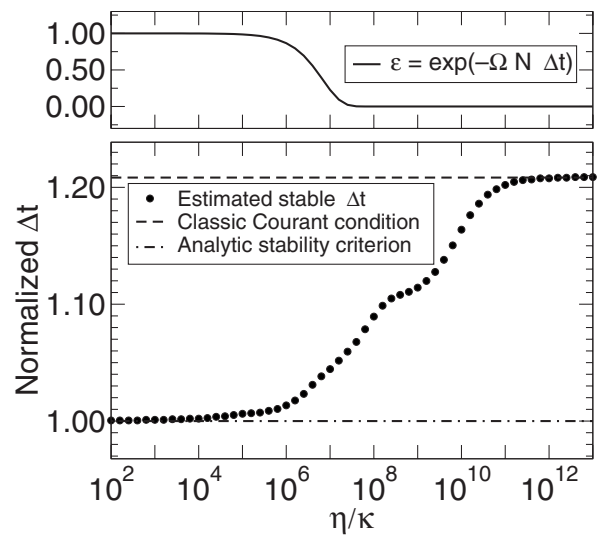

Figure 4. Numerical determination of the stability criterion plotted as a function of $\eta / k_{0}$ with the number of memory points $N=20$. The estimate is made by varying $\Delta t$ for a given value of $\eta / k_{0}$ and keeping the other parameters fixed. Below the black dots, the scheme is stable; above the black dots, it is unstable. The key result is that the stability criterion is bounded between the classic Courant condition of equation 49 as $\eta / k_{0} \rightarrow 0$ and the analytic criterion of equation 48 as $\eta / k_{0} \rightarrow \infty$. The specific shape of the black dotted curve can vary somewhat depending on the values of the other parameters; however, it always stays between the upper and lower limits just mentioned. 
of the scheme by comparing numerical results for the velocity dispersion and attenuation to exact analytic results.

\section{Snapshots}

We now consider a modeling example that requires the complete convolution form of the dynamic-permeability operator.

Consider the situation depicted in Figure 5 involving a compressional point source that sends out both fast and slow compressional waves. The center frequency of the compressional pulse is $50 \mathrm{kHz}$. The dimensions of the numerical modeling domain (roughly $1 \mathrm{~m}$ to each side) and the frequency of the source are typical of some laboratory experiments on ocean sediments performed by Hefner and Williams (2006) and of the underwater field experiments on ocean sediments performed by Williams et al. (2002).

To obtain nontrivial results involving the reflection and transmission of both fast and slow waves, we introduce a permeability interface below the source point (denoted with a horizontal dashed line in the figure) while keeping all other material properties uniform throughout the modeled region. Above the interface, the permeability is $k_{0}=20$ darcy; below, it is 0.2 darcy. This results in the Biot relaxation frequency being $1.1 \mathrm{kHz}$ above the interface and $110 \mathrm{kHz}$ below. Thus, for waves above the interface, the $50-\mathrm{kHz}$ pulse is in the high-frequency domain where the slow wave is propagatory, whereas below the interface, the pulse is in the low-frequency domain where the slow wave is purely diffusive. The two columns on the left in Figure 5 are plotted using the full scale of the pressure pulses, and the columns on the right are plotted using a saturated scale that allows the smaller amplitude details of the slow waves to be observed.

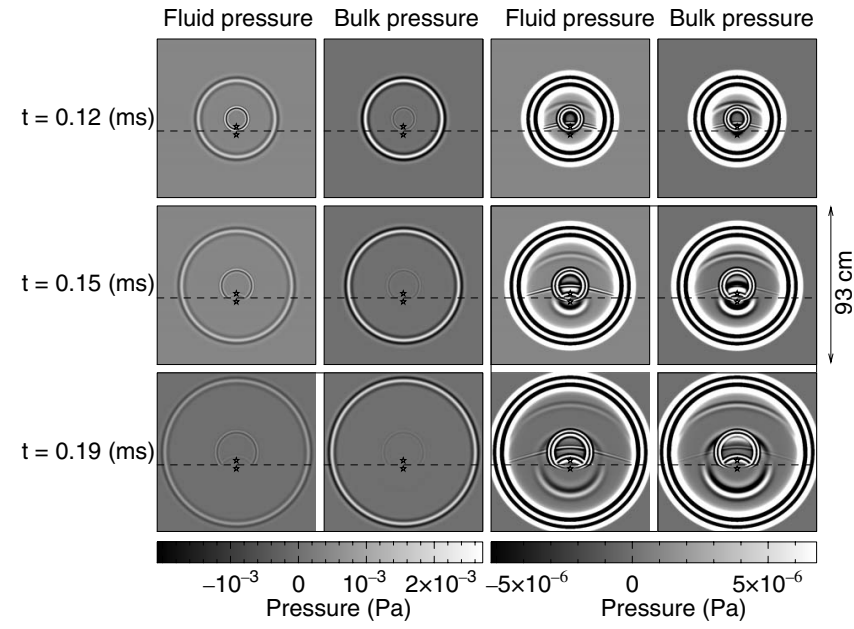

Figure 5. Snapshots of the fluid pressure (first and third columns) and bulk pressure (second and fourth columns) for a point source at the center generating a 50-kHz central-frequency compressional pulse. The two columns on the left are plotted at full scale, and the columns on the right are plotted with a saturated scale that allows the fine details of the slow waves to be observed. In this example, all material properties except permeability are uniform throughout. Above the dashed line, $k_{0}=2 \times 10^{-11} \mathrm{~m}^{2}$ (20 darcy), the relaxation frequency is $1.1 \mathrm{kHz}$, and the wave propagation is thus in the high-frequency regime where the slow wave is propagatory. Below the dashed line, $k_{0}=2 \times 10^{-13} \mathrm{~m}^{2}(0.2$ darcy), the relaxation frequency is $110 \mathrm{kHz}$, and the wave propagation is in the low-frequency regime where the slow wave is purely diffusive. The various waves are the primary or reflected/transmitted fast and slow waves. The stars indicate the positions where waveforms are recorded (see Figure 6).
When the direct, fast $\mathrm{P}$-wave arrives at the permeability interface, most of its energy is transmitted downward; however, a very weak reflected $\mathrm{P}$-wave and a somewhat stronger reflected slow wave are observed as well. When the direct slow wave arrives at the interface, there are generated weakly transmitted and reflected fast P-waves as well as a strongly reflected slow wave. The slow wave that is transmitted is a pure diffusion. No shear waves are generated at the source. The shear waves generated by compressional pulses at a contrast in permeability are much smaller in energetic amplitude than the various compressional pulses and are not observed in the present plots because a shear wave propagates with no change in either fluid or bulk pressure.

Because the interface separates wave propagation in the low- and high-frequency regimes, proper modeling of the slow-wave amplitudes in this example requires the inclusion of the convolution integral involving the Darcy flow. If only the low-frequency form of the generalized Darcy's law (equation 7) is used as opposed to the complete convolution of the present example, the amplitudes of the slow waves are far too large, as is seen in the traces of Figure 6. In this figure, the column to the left corresponds to a receiver located just above the interface (denoted with a star in Figure 5), and the column
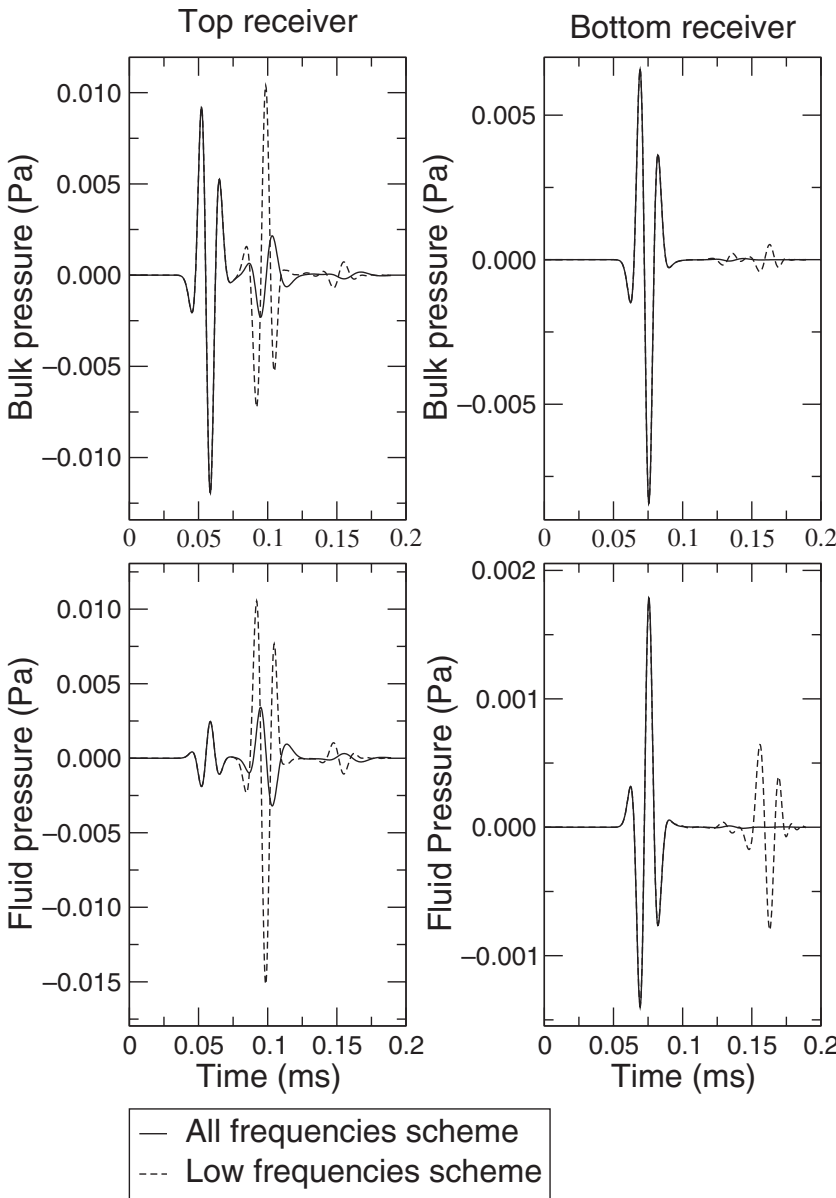

Figure 6. Waveforms recorded at the starred positions in Figure 5. The solid lines are the result of the present study's modeling that includes the dynamic-permeability convolution, whereas the dashed lines are the result of the low-frequency modeling in which the permeability coefficient is taken as a simple multiplicative constant. The main difference is that the low-frequency equations grossly underestimate the attenuation and dispersion of the slow waves. 
to the right corresponds to a receiver just below the interface. The reason the low-frequency equations predict a slow wave recorded at the lower receiver with such large amplitude is because, as the slow

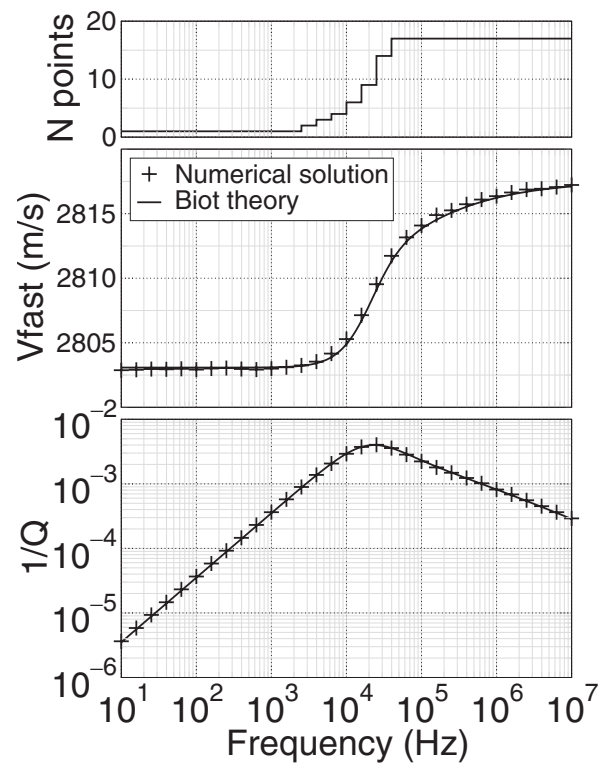

Figure 7. Demonstration of the accuracy of the scheme for Biot fast waves. The fast-wave velocity and attenuation is determined by performing a transmission experiment at the various center frequencies, as denoted with crosses. The number of memory points used in the convolution is given in the top part. The solid lines in the two lower parts are the analytically exact results.

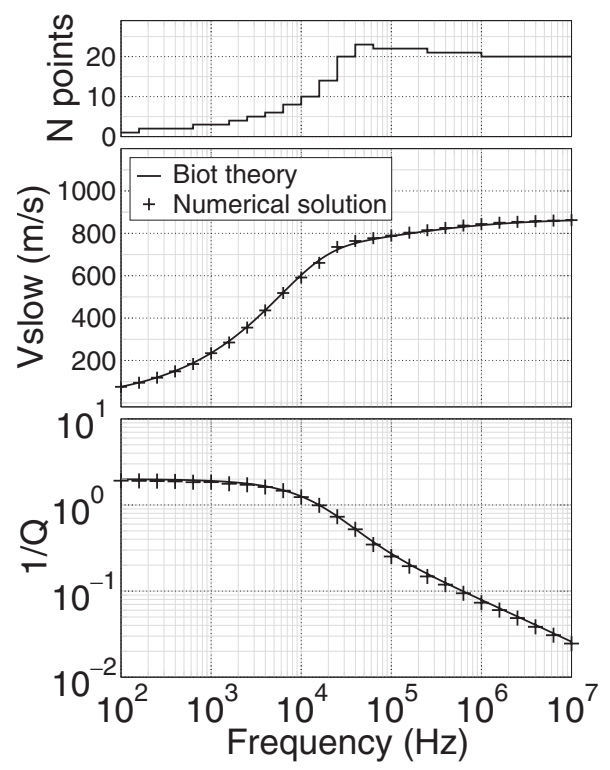

Figure 8. Demonstration of the accuracy of the scheme for Biot slow waves. The slow-wave velocity and attenuation is determined by performing a transmission experiment at the various center frequencies, as denoted with crosses. The number of memory points used in the convolution is given in the top part. The solid lines in the two lower parts are the analytically exact results.
Table 1. Material properties of a lightly consolidated sand.

Solid grain material

$\begin{array}{ll}\text { Bulk modulus }\left(K_{s}\right) & 36.0 \mathrm{GPa} \\ \text { Density }(\rho) & 2650 \mathrm{~kg} / \mathrm{m}^{3}\end{array}$

Skeletal framework of grains

$\begin{array}{ll}\text { Bulk modulus }\left(K_{d}\right) & 621 \mathrm{MPa} \\ \text { Shear modulus }(\mu) & 455 \mathrm{MPa} \\ \text { Porosity }(\phi) & 0.3 \\ \text { Permeability }(k) & 10^{-12} \mathrm{~m}^{2}\end{array}$

Fluid

Bulk modulus $\left(K_{f}\right) \quad 2.25 \mathrm{GPa}$

Density $\left(\rho_{f}\right) \quad 1000 \mathrm{~kg} / \mathrm{m}^{3}$

Viscosity $(\eta) \quad 10^{-3} \mathrm{~N} \mathrm{~s} \mathrm{~m}^{-2}$

wave passed from the source to the interface, the attenuation was being greatly underestimated. This example demonstrates the importance of using the complete theory involving the dynamic-permeability convolution.

\section{Dispersion and attenuation in a homogeneous material}

To quantify the accuracy of the present finite-difference modeling, the velocity dispersion and attenuation of both fast and slow waves is determined as a function of frequency and compared to the exact analytic results in Figures 7 and 8.

The numerical experiments are performed by sending a plane wave across a uniform region (properties are given in Table 1). Each data point given in Figures 7 and 8 corresponds to a different experiment involving a pulse with a different center frequency. A Morlet wavelet is used having a narrow band of support around a center frequency. By recording the solid particle velocity at two points in the direction of propagation, and time integrating the recording to obtain the maximum displacement amplitude and associated traveltime for each recording, the velocity and attenuation are determined at each frequency. Upon comparing the crosses (finite-difference results) to the solid line (analytic results) in Figures 7 and 8, the scheme is seen to produce accurate results. If only the low-frequency form of the generalized Darcy's law (equation 7) is used, the attenuation falls off far too rapidly as $\omega^{-1}$ instead of as $\omega^{-1 / 2}$, as seen in the figure.

\section{CONCLUSION}

A time-domain finite-difference scheme was presented for solving Biot's equations across all frequencies while allowing for the possible development of viscous boundary layers in the pores at sufficiently high frequencies. In this case, the generalized Darcy law controlling the movement of fluid relative to solid contains a time convolution between a kernel that exponentially decays into the past and the past time values of the Darcy flow. It was shown how to model this convolution in an efficient and accurate manner that typically does not require more than 20 past time values to be stored (and often considerably fewer). Snapshots generated by the scheme show how slow waves above the viscous-boundary-layer transition frequency have a propagatory nature to them, whereas slow waves below the 
transition frequency are pure diffusions. Getting the amplitudes of these slow waves correct requires the use of the complete theory involving the dynamic-permeability convolution. The accuracy of the scheme was determined by comparing the attenuation and velocity of numerically modeled plane waves at different frequencies to the analytic results. The accuracy was excellent over a broad range of frequencies that included the transition frequency.

\section{ACKNOWLEDGMENTS}

Work of SRP was performed under the auspices of the U. S. Department of Energy by the University of California, Lawrence Berkeley National Laboratory, under Contract No. DE-AC02$05 \mathrm{CH} 11231$ and supported specifically by the Geosciences Research Program of the U. S. Department of Energy, Office of Basic Energy Sciences, Division of Chemical Sciences, Geosciences and Biosciences

\section{REFERENCES}

Biot, M. A., 1956, Theory of propagation of elastic waves in a fluid-saturated porous solid: 1 - Low-frequency range: Journal of the Acoustical Society of America, 28, 168-178.

Biot, M. A., and D. G. Willis, 1957, The elastic coefficients of the theory of consolidation: Journal of Applied Mechanics, 24, 594-601.

Carcione, J. M., 1996, Wave propagation in anisotropic, saturated porous media: Plane-wave theory and numerical simulation: Journal of the Acoustical Society of America, 99, 2655-2666.

Carcione, J. M., and H. H. Helle, 1999, Numerical solution of the poroviscoelastic wave equation on a staggered mesh: Journal of Computational Physics, 154, 520-527.

Carcione, J. M., and G. Quiroga-Goode, 1995, Some aspects of the physics and numerical modeling of Biot compressional waves: Journal of Computational Acoustics, 3, 261-272.
Hanyga, A., and J.-F. Lu, 2005, Wave field simulation for heterogeneous transversely isotropic porous media with the JKD dynamic permeability: Computational Mechanics, 36, 196-208.

Hefner, B. T., and K. L. Williams, 2006, Sound speed and attenuation measurements in unconsolidated glass-bead sediments saturated with viscous pore fluids: Journal of the Acoustical Society of America, 120, $2538-2549$.

Johnson, D. L., J. Koplik, and R. Dashen, 1987, Theory of dynamic permeability and tortuosity in fluid-saturated porous media: Journal of Fluid Mechanics, 176, 379-402.

Levander, A. R., 1988, Fourth-order finite-difference P-SV seismograms: Geophysics, 53, 1425-1436.

Madariaga, R., 1976, Dynamics of an expanding circular fault: Bulletin of the Seismological Society of America, 66, 163-182.

Masson, Y. J., S. R. Pride, and K. T. Nihei, 2006, Finite-difference modeling of Biot's poroelastic equations at seismic frequencies: Journal of Geophysical Research, 111, B10305.

Özdenvar, T., and G. A. McMechan, 1997, Algorithms for staggered-grid computations for poroelastic, elastic, acoustic, and scalar wave equations Geophysical Prospecting, 45, 403-420.

Plyushchenkov, B. D., and V. Turchaninov, 2000, Acoustic logging modeling by refined Biot's equations: International Journal of Modern Physics C, 12, 305-396.

Pride, S. R., 1994, Governing equations for the coupled electromagnetics and acoustics of porous media: Physical Review B, 50, 15678-15696.

, 2005, Relationships between seismic and hydrological properties, in Y. Rubin and S. S. Hubbard, eds., Hydrogeophysics: Springer, 253-291.

Virieux, J., 1986, P-SV wave propagation in heterogeneous media: Velocitystress finite-difference method: Geophysics, 51, 889-901.

Virieux, J., and R. Madariaga, 1982, Dynamic faulting studied by a finite-difference method: Bulletin of the Seismological Society of America, 72 , $345-369$.

Williams, K. L., D. R. Jackson, E. I. Thorsos, D. Tang, and S. G. Schock, 2002, Comparison of sound speed and attenuation measured in a sandy sediment to predictions based on the Biot theory of porous media: IEEE Journal of Oceanic Engineering, 27, 413-428.

Zhang, J., 1999, Quadrangle-grid velocity-stress finite difference method for poroelastic wave equations: Geophysical Journal International, 139, $171-182$.

Zhu, X., and G. A. McMechan, 1991, Numerical simulation of seismic responses of poroelastic reservoirs using Biot theory: Geophysics, 56, 328-339. 